\title{
Dibaryons with Strangeness: their Weak Nonleptonic Decay using SU(3) Symmetry and how to find them in Relativistic Heavy-Ion Collisions
}

\author{
Jürgen Schaffner-Bielich \\ RIKEN BNL Research Center, Brookhaven National Laboratory, Upton, New York 11973-5000, USA \\ Raffaele Mattiello \\ The Niels Bohr Institute, Blegdamsvej 17, DK-2100 Copenhagen, Denmark \\ Heinz Sorge \\ Department of Physics $\& 3$ Astronomy, State University of New York at Stony Brook, NY 11794-3800, USA
}

(September 17, 2018)

Weak $\mathrm{SU}(3)$ symmetry is successfully applied to the weak hadronic decay amplitudes of octet hyperons. Weak nonmesonic and mesonic decays of various dibaryons with strangeness, their dominant decay modes, and lifetimes are calculated. Production estimates for BNL's Relativistic Heavy-Ion Collider are presented employing wave function coalescence. Signals for detecting strange dibaryon states in heavy-ion collisions and revealing information about the unknown hyperon-hyperon interactions are outlined.

25.75.-q, 13.30.Eg, 14.20.Pt

Relativistic heavy-ion collisions provide a prolific source of strangeness: dozens of hyperons and kaons are produced in central collisions at BNL's AGS and at CERN SPS (see e.g. [1]). This opens the exciting perspective of forming composites with multiple units of strangeness hitherto unachievable with conventional methods.

Exotic forms of deeply bound objects with strangeness have been proposed by Bodmer [2] as collapsed states of matter, either consisting of baryons or quarks. A six-quark bag state, the $\mathrm{H}$ dibaryon, was predicted by Jaffe [3]. Other bound dibaryon states with strangeness were proposed using quark potentials [4, 4 , or the Skyrme model [6]. On the hadronic side, hypernuclei are known to exist already for a long time. The double $\Lambda$ hypernuclear events reported so far are closely related to the $\mathrm{H}$ dibaryon [7]. Metastable exotic multihypernuclear objects (MEMOs) as well as purely hyperonic systems of $\Lambda$ 's and $\Xi$ 's were introduced in [8.9] as the hadronic counterparts to multistrange quark bags (strangelets) [10,11. Most recently, the Nijmegen soft-core potential was extended to the full baryon octet and bound states of $\Sigma \Sigma$, $\Sigma \Xi$, and $\Xi \Xi$ dibaryons were predicted [12].

One major uncertainty for the detection of such speculative states is their (meta)stability. MEMOs, for example, consists of nucleons, $\Lambda$ 's, and $\Xi$ and are metastable by virtue of Pauli-blocking effects. Only two investigations about the weak decay of dibaryons exist so far: In [13], the $\mathrm{H}$ dibaryon was found to decay dominantly by $\mathrm{H} \rightarrow \Sigma^{-}+p$ for moderate binding energies. The $(\Lambda \Lambda)_{b}$, which has exactly the same quantum numbers as the $\mathrm{H}$ dibaryon, was studied in [14]. Here, the main nonmesonic channel was found to be $(\Lambda \Lambda)_{b} \rightarrow \Lambda+n$.

In the following, we will revive an 'old' approach to calculate weak decay channels and lifetimes of various strange dibaryons using $\mathrm{SU}(3)$ symmetric contact interactions. Finally, we present production estimates for RHIC combining transport simulations using Relativistic Quantum Molecular Dynamics, which is widely used for simulations of relativistic heavy ion collisions, with wave function coalescence.

The weak decays of the octet hyperons $(\Lambda, \Sigma$, and $\Xi)$ can be described by an effective $\mathrm{SU}(3)$ symmetric interaction with a parity-violating $(A)$ and a parity conserving $(B)$ amplitude [15]. The weak operator is assumed to be proportional to the Gell-Mann matrix $\lambda_{6}$ which ensures hypercharge violation $|\Delta Y|=1$, the $\Delta I=1 / 2$ rule and the Lee-Sugawara relation for the $A$ amplitudes. There are three $\mathcal{C P}$ invariant terms for the $A$ amplitude. One contributes to $\Sigma^{+} \rightarrow n+\pi^{+}$and can be ignored. The two remaining parameters can be well fitted to the experimental data (see below).

The problem is to describe correctly the $B$ amplitudes which defy a consistent explanation. Traditionally, one uses the pole model which in its basic version is not able to describe the experimental measured amplitudes [16]. Various solutions have been proposed to remedy the situation like including the vector meson pole 17] or hyperon resonances 18]. On the other hand, as pointed out in [16], there is no serious consideration about a contact interaction for the $B$ amplitudes in the literature.

General SU(3) symmetry and $\mathcal{C P}$ invariance results in five independent terms for the $B$ amplitudes 15. We find that one term gives the wrong sign either to the $B$ amplitudes for the $\Lambda$ or for the $\Xi$ 's. Hence, it must be small compared to the others. Another term gives a contribution to $\Sigma^{-} \rightarrow n+\pi^{-}$and can be neglected. Only three terms remain with coupling constants to be adjusted to the seven measured $B$ amplitudes.

The corresponding Lagrangian for both amplitudes reads

$$
\mathcal{L}=D \operatorname{Tr} \bar{B} B\left[P, \lambda_{6}\right]+F \operatorname{Tr} \bar{B}\left[P, \lambda_{6}\right] B+G \operatorname{Tr} \bar{B} P \gamma_{5} B \lambda_{6}
$$




\begin{tabular}{c|r|r|c|r}
\hline \hline & \multicolumn{2}{|c|}{$\mathrm{A}$} & \multicolumn{2}{c}{$\mathrm{B}$} \\
& $\exp$ & $\mathrm{SU}(3)$ & $\exp$ & $\mathrm{SU}(3)$ \\
\hline$\Lambda \rightarrow p+\pi^{-}$ & 3.25 & 3.25 & 22.1 & 22.1 \\
$\Lambda \rightarrow n+\pi^{0}$ & -2.37 & -2.30 & -16.0 & -15.6 \\
$\Sigma^{+} \rightarrow n+\pi^{+}$ & 0.13 & 0.0 & 42.2 & 40.0 \\
$\Sigma^{+} \rightarrow p+\pi^{0}$ & -3.27 & -3.33 & 26.6 & 28.3 \\
$\Sigma^{-} \rightarrow n+\pi^{-}$ & 4.27 & 4.71 & -1.44 & 0.0 \\
$\Xi^{0} \rightarrow \Lambda+\pi^{0}$ & 3.43 & 3.19 & -12.3 & -11.7 \\
$\Xi^{-} \rightarrow \Lambda+\pi^{-}$ & -4.51 & -4.51 & 16.6 & 16.6 \\
\hline \hline
\end{tabular}

TABLE I. The hyperon weak decay amplitudes in $\mathrm{SU}(3)_{\text {weak }}$ compared to experimental data taken from 16 . All values are in units of $10^{-7}$.

$$
+H \operatorname{Tr} \bar{B} \lambda_{6} \gamma_{5} B P+J \operatorname{Tr} \bar{B}\left\{P, \lambda_{6}\right\} \gamma_{5} B
$$

$B$ stands for the baryon octet and $P$ for the pseudoscalar nonet. The choice $D=4.72$ and $F=-1.62$ for the $A$ amplitudes and $G=40.0, H=47.8$, and $J=-7.1$ for the $B$ amplitudes in units of $10^{-7}$ gives a good agreement with the experimental data as shown in Table I. We point out that the $B$ amplitudes do not follow a Lee-Sugawara relation 15]. Using this model for the weak hyperon decay, one can calculate the weak mesonic and nonmesonic decay of strange dibaryons using a Hulthen-like wave function [14]. The meson exchange model for the weak nonmesonic decay of hypernuclei has been proven to be quite successful [19]. We include pion and kaon exchange in our model for the nonmesonic decay as they are the dominant contributions. Effects from short-range contributions like vector meson exchange [19] and direct quark-quark contributions 20] have been found to be less important. We find that the p-wave contributions originating from the $B$ amplitudes, the kaon exchange terms and the interference terms are particularly important for the nonmesonic decay channels. Hence, a consistent scheme of both amplitudes turns out to be a crucial ingredience. Clearly, a more fundamental approach is desirable but is at present not at hand before we understand strong interactions at the confinement scale.

For a detection in heavy-ion experiments we are mainly interested in candidates whose final decay products are charged:

$$
\begin{aligned}
\left(\Sigma^{+} p\right)_{b} & \rightarrow p+p \\
\left(\Xi^{0} p\right)_{b} & \rightarrow p+\Lambda \\
\left(\Xi^{0} \Lambda\right)_{b} & \rightarrow p+\Xi^{-} \text {or } \Lambda+\Lambda \\
\left(\Xi^{0} \Xi^{-}\right)_{b} & \rightarrow \Xi^{-}+\Lambda .
\end{aligned}
$$

We find that the decay lengths for all of the above strange dibaryons is between $c \tau \approx 1-5 \mathrm{~cm}$. Fig. 1 1 shows the calculated branching ratios as a function of the binding energy.

(a): There is only one nonmesonic decay channel for $(\Sigma p)_{b} \rightarrow p+p$ which we find to be dominant above 5 $\mathrm{MeV}$ binding energy. The dibaryon should show up in the

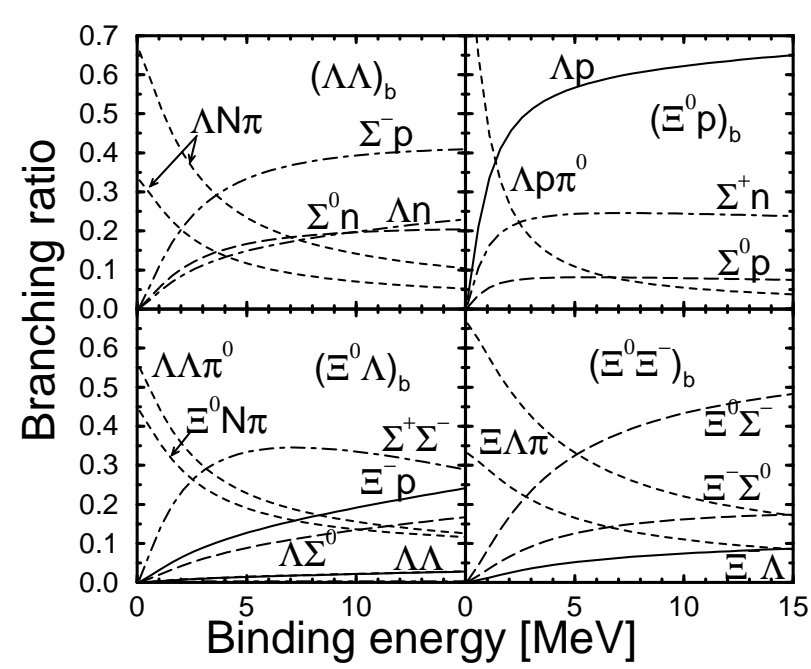

FIG. 1. Weak decay branching ratios for several strange dibaryons versus the binding energy. Solid lines denote ultimately charged final states, while dash-dotted lines indicate final states accessible with a neutron detector.

invariant $p p$ mass spectrum after background subtraction from event-mixing at $M=2.128 \mathrm{GeV}-\epsilon$ where $\epsilon$ is the binding energy. With this method the weak decay of the lightest hypernucleus ${ }_{\Lambda}^{3} \mathrm{H} \rightarrow{ }^{3} \mathrm{He}+\pi^{-}$has been detected in heavy-ion collisions by the E864 collaboration [21].

(b): For the $\left(\Xi^{0} p\right)_{b}$ bound state only one mesonic but three different nonmesonic channels contribute. The dominant nonmesonic decay turns out to be $\left(\Xi^{0} p\right)_{b} \rightarrow$ $\Lambda+p$ already for a binding energy of $2 \mathrm{MeV}$ or more. The decay itself resembles the one for the weak decay of the $\Xi^{-}$or $\Omega^{-}$, which have already been detected by several experiments (see contributions in [1]). Instead of an outgoing $\pi^{-}$or $K^{-}$there is a proton leaving the first weak vertex.

(c): The dibaryon $\left(\Xi^{0} \Lambda\right)_{b}$ decays to $\Xi^{-}+p$ and, with a small fraction, to two $\Lambda$ 's. Therefore, it can be seen in $\Xi^{-} p$ or $\Lambda \Lambda$ invariant mass plots. One has indeed seen two-Lambda events at the AGS by experiment E896 22] and experiment WA97 at the SPS has already published two-Lambda correlation functions 23. There are plans to study the correlation of two $\Lambda$ 's on an event-by-event basis at the STAR detector at BNL's RHIC 24.

(d): The $\left(\Xi^{0} \Xi^{-}\right)_{b}$ dibaryon has been predicted to be bound [12] and its decay to $\Xi^{-}+\Lambda$ has a branching ratio of a few percent.

The other bound candidates predicted by the Nijmegen model 12] involve weak decays with $\Sigma$ hyperons in the final state. If one can measure neutrons, one is sensitive to all proposed states:

$$
\begin{aligned}
& \left(\Sigma^{-} \Sigma^{-}\right)_{b} \rightarrow \Sigma^{-}+n+\pi^{-} \\
& \left(\Sigma^{+} \Sigma^{+}\right)_{b} \rightarrow \Sigma^{+}+p \\
& \left(\Xi^{0} \Sigma^{+}\right)_{b} \rightarrow \Sigma^{+}+\Lambda \\
& \left(\Xi^{-} \Sigma^{-}\right)_{b} \rightarrow \Sigma^{-}+\Sigma^{-}
\end{aligned}
$$




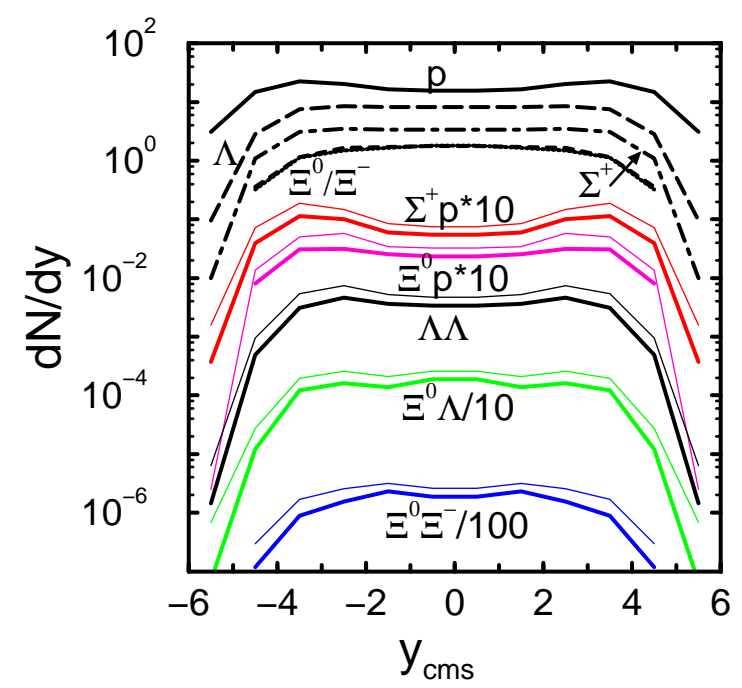

FIG. 2. Rapidity distribution of baryons (upper curves) and strange dibaryons (lower curves) using RQMD2.4 with wavefunction coalescence for $\mathrm{Au}+\mathrm{Au}$ collisions at $\sqrt{s}=200$ $\mathrm{AGeV}$. Upper curves are for a binding energy of $E_{b}=5 \mathrm{MeV}$, lower ones for $E_{b}=1 \mathrm{MeV}(\alpha=2)$.

$$
\begin{gathered}
\left(\Xi^{0} \Xi^{0}\right)_{b} \rightarrow \Sigma^{+}+\Xi^{-} \\
\left(\Xi^{-} \Xi^{-}\right)_{b} \rightarrow \Sigma^{-}+\Xi^{-} .
\end{gathered}
$$

In addition, one can see the nonmesonic decay involving a direct neutron in the final state, like $(\Lambda \Lambda)_{b} \rightarrow \Lambda+n$ and $\left(\Xi^{-} \Lambda\right)_{b} \rightarrow \Xi^{-}+n$. Thus, a possible $\Lambda n$ or $\Xi^{-} n$ invariant mass distribution might reveal important information about the unknown hyperon-hyperon interactions hitherto unaccessible by experiment. We find that the dominant nonmesonic decay for $(\Lambda \Lambda)_{b}$ is the same as for the $\mathrm{H}$ dibaryon, i.e. $(\Lambda \Lambda)_{b} \rightarrow \Sigma^{-}+p$. This means that the two dibaryons are indistinguishable experimentally. Note, that the nonmesonic decay of the $(\Lambda \Lambda)_{b}$ always involves a neutral particle in the final state. Searches for the $\mathrm{H}$ dibaryon in heavy-ion collisions are indeed sensitive for a weak decay with a $\Sigma^{-}$in the final state 25] and may be utilized to look for other exotic candidates. Especially the weak decay (3b) looks very similar to the weak decay of the $\mathrm{H}$ dibaryon one is already looking for, but with the opposite sign for the $\Sigma$ hyperon.

Let us now focus on formation probabilities for strange baryon clusters $\Lambda \Lambda, p \Sigma^{+}, p \Xi^{0}, \Xi^{0} \Lambda$ and $\Xi^{0} \Xi^{-}$. The coalescence model provides estimates by simple phasespace arguments. Momentum coalescence has been successful in describing data at low energies (see e.g. [26]). At relativistic bombarding energies, however, expansion of the source and collective flow have been shown to strongly modify the production rates [27,28]. Therefore, we will combine source distributions for baryons borrowed from microscopic transport calculations [29] with a coalescence prescription in phase space as detailed in [30]. This procedure has been successful to describe deuteron yields, momentum distributions [31,30] and is in accord with studies of proton-deuteron correlations [32]. Assuming uncorrelated emission the formation rate can be expressed as

$$
\begin{aligned}
& \frac{d N}{d \vec{P}}=g \int f_{\mathrm{A}}\left(\vec{x}_{1}, \vec{p}_{1}\right) f_{\mathrm{B}}\left(\vec{x}_{2}, \vec{p}_{2}\right) \rho_{\mathrm{AB}}(\Delta \vec{x}, \Delta \vec{p}) \\
& \delta\left(\vec{P}-\vec{p}_{1}-\vec{p}_{2}\right) d^{3} x_{1} d^{3} x_{2} d^{3} p_{1} d^{3} p_{2}
\end{aligned}
$$

where $\Delta \vec{x}=\vec{x}_{1}-\vec{x}_{2}$ and $\Delta \vec{p}=\left(\vec{p}_{1}-\vec{p}_{2}\right) / 2$ are given in the respective 2 -body cms (i.e. $\vec{P} \equiv 0$ ). One has to multiply the rate with a symmetry factor of $1 / 2$, if the outgoing particles are identical. For the wave function we assume a Hulthen-shape as for the calculations of weak decay properties $\Psi(r)=c / r\left(\mathrm{e}^{-\kappa r}-\mathrm{e}^{-\alpha \kappa r}\right)$. The statistical prefactors $g$ account for the lack of information about 2-body correlations with respect to internal degrees of freedom. It includes spin average and the projection on one particular final isospin state of the dibaryon. All strange dibaryons are assumed to be formed in spinsinglet states. The reduction to the correct 'number' of possible quantum states depends crucially on the assumption of uncorrelated emission and the nature of the bound state. Since the multiparticle correlations during the break up are not well known we consider the g-values as estimates which need further guidance - and insight. The predictions for strange dibaryons are depicted in Fig. 2. Variations in the wave function parameters $E_{b}=1-20$ $\mathrm{MeV}\left(\kappa=\sqrt{2 \mu E_{b}}\right)$ and $\alpha=2-6$ lead only to minor changes in the final result $( \pm 20 \%)$. Therefore, we have chosen to present calculations for the two most extreme parameter-sets $\left(E_{b} \approx 5 \mathrm{MeV}, \alpha=2\right)$ and $\left(E_{b} \approx 1\right.$ $\mathrm{MeV}, \alpha=2)$. The formation of $\Lambda \Lambda$-states and deuterons (see also [33, 34]) is diminished by the volume expansion close to midrapidity. For nucleon-hyperon bound states the rapidity shift towards projectile and target is somewhat stronger due to enhanced nuclear freeze-out densities at forward/backward rapidities. Note, that strange dibaryons produced at these rapidities have substantially longer decay lengths which opens the possibility of detecting them at small forward or backward angles. The B-parameter $B_{\mathrm{AB}} \propto \frac{1}{g_{\mathrm{AB}}} N(\mathrm{~A}, \mathrm{~B}) / N(\mathrm{~A}) N(\mathrm{~B})$ measuring the production rates of dibaryons increases by a factor of two to three comparing $\Lambda \Lambda$ and $\Xi \Xi$-states. This enhancement is compatible with an 'early' freeze-out scenario for multiple strange baryons as argued in [35]: Clusters with high strangeness might be formed more likely, as they decouple earlier from the collisions zone.

There are several searches in heavy-ion collisions for the $\mathrm{H}$ dibaryon [36,25] and for long-lived strangelets [37,38 with high sensitivities. Hypernuclei have been detected most recently in heavy-ion reactions at the AGS by the E864 collaboration [21]. The dibaryon states studied here are short-lived. They can in principle be detected in present and future experiments by the following means:

1) Experiments with a time-projection chamber can track for unique exotic decays like a charged particle de- 
caying to two charged particles or tracks forming a vertex a few cm outside the target.

2) Experiments sensitive to hyperons can look for peaks in the invariant mass spectrum of $p p, p \Lambda, \Lambda \Lambda, p \Xi^{-}$, and $\Lambda \Xi^{-}$by background subtraction using event mixing.

3) Resonances (unbound states) can be seen in the correlation function of $\Lambda \Lambda$ [39] and $\Lambda \Xi^{-}$. Two-particle interferometry is a powerful tool to extract information about their (unknown) strong interaction potential as the correlation function depends sensitively on final-state interactions [40]. The Coulomb potential does not mask the strong interactions at low momenta as pointed out in 41] for $\Lambda p$ so that information about the presently unknown hyperon-hyperon forces can be extracted as shown in 42 ).

The STAR experiment at the BNL's Collider RHIC is able to detect short-lived candidates as well as exotic resonances 43,44. One $(\Lambda \Lambda)$ resonance can be seen out of 100 uncorrelated $\Lambda$ 's 43]. For the production rates given in Fig. 2 and $10^{6}$ central events, even the bound $\left(\Xi^{0} \Xi^{-}\right)_{b}$ dibaryon can be seen by backtracking for a reconstruction efficiency of only .2\% or better which is indeed feasible for lifetimes around $10^{-10} \mathrm{~s}[43$.

In this paper we have calculated production rates of strange dibaryons via the coalescence mechanism of independently produced baryons. Finally, we want to point out that another mechanism for their formation might be possible. Via the separation and distillation process, a hot quark-gluon plasma gets enriched with strangeness [45] leading to strangelet creation. If strangelets are unstable they can form a doorway state by decaying to strange dibaryons and increase the production rates.

We are pleased to acknowledge helpful discussions with Ken Barish, Hank Crawford, Carsten Greiner, Huan Huang, Sonja Kabana, Richard Majka, Jamie Nagle, Grazyna Odyniec, Klaus Pretzl, Gulshan Rai, Jack Sandweiss, and Horst Stöcker.

[1] International Symposium on Strangeness in Quark Matter 1998, J. Phys. G 25, 1 (1999).

[2] A. R. Bodmer, Phys. Rev. D 4, 1601 (1971).

[3] R. L. Jaffe, Phys. Rev. Lett. 38, 195 (1977).

[4] T. Goldman, K. Maltman, G. J. Stephenson, and F. Wang, Phys. Rev. Lett. 59, 627 (1987).

[5] T. Goldman, K. Maltman, G. J. Stephenson, J.-L. Ping, and F. Wang, Mod. Phys. A 13, 59 (1998).

[6] B. Schwesinger, F. G. Scholtz, and H. B. Geyer, Phys. Rev. D 51, 1228 (1995).

[7] R. H. Dalitz, D. H. Davis, P. H. Fowler, A. Montwill, J. Pniewski, and J. A. Zakrzewski, Proc. Roy. Soc. Lond. A426, 1 (1989).

[8] J. Schaffner, C. Greiner, and H. Stöcker, Phys. Rev. C 46, 322 (1992).
[9] J. Schaffner, C. B. Dover, A. Gal, C. Greiner, and H. Stöcker, Phys. Rev. Lett. 71, 1328 (1993).

[10] E. P. Gilson and R. L. Jaffe, Phys. Rev. Lett. 71, 332 (1993).

[11] J. Schaffner-Bielich, A. Diener, C. Greiner, and H. Stöcker, Phys. Rev. C 55, 3038 (1997).

[12] V. G. J. Stoks and T. A. Rijken, Phys. Rev. C 59, 3009 (1999).

[13] J. F. Donoghue, E. Golowich, and B. R. Holstein, Phys. Rev. D 34, 3434 (1986).

[14] M. I. Krivoruchenko and M. G. Shchepkin, Sov. J. Nucl. Phys. 36, 769 (1982).

[15] R. E. Marshak, Riazuddin, and C. P. Ryan, Theory of weak interactions in particle physics (Wiley-Interscience, New York, 1969).

[16] J. F. Donoghue, E. Golowich, and B. R. Holstein, Phys. Rep. 131, 319 (1986).

[17] M. Gronau, Phys. Rev. D 5, 118 (1972).

[18] A. L. Yaouanc, O. Pene, J. C. Raynal, and L. Oliver, Nucl. Phys. B 149, 321 (1979).

[19] A. Parreno, A. Ramos, and C. Bennhold, Phys. Rev. C 56, 339 (1997).

[20] M. Oka, K. Sasaki, and T. Inoue, nucl-th/9906042.

[21] L. E. Finch et al. (E864 collaboration), Nucl. Phys. A661, 395c (1999).

[22] H. Caines et al. (E896 collaboration), Nucl. Phys. A661, 170c (1999).

[23] A. Jacholkowski et al., J. Phys. G 25, 423 (1999).

[24] R. Bellwied, J. Phys. G 25, 437 (1999).

[25] H. J. Crawford, Nucl. Phys. A639, 417c (1998).

[26] L. P. Csernai and J. I. Kapusta, Phys. Rep. 131, 223 (1986).

[27] R. Mattiello, A. Jahns, H. Sorge, H. Stoecker, and W. Greiner, Phys. Rev. Lett. 74, 2180 (1995).

[28] J. Barrette et al., nucl-ex/9906005.

[29] H. Sorge, Phys. Rev. C 52, 3291 (1995).

[30] R. Mattiello, H. Sorge, H. Stoecker, and W. Greiner, Phys. Rev. C 55, 1443 (1997).

[31] J. Nagle, S. Kumar, D. Kusnezov, H. Sorge, and R. Mattiello, Phys. Rev. C 53, 367 (1996).

[32] S. Panitkin, (E877 Coll.), Proc. Int. Conf. Heavy Ion Physics at the AGS '96, eds. C. A. Pruneau et al., WSUNP-96-16, 147 (1996).

[33] H. Sorge, J. L. Nagle, and B. S. Kumar, Phys. Lett. B 355, 27 (1995).

[34] B. Monreal et al., Phys. Rev. C 60, 031901 (1999).

[35] H. van Hecke, H. Sorge, and N. Xu, Phys. Rev. Lett. 81, 5764 (1998).

[36] J. Belz et al., Phys. Rev. Lett. 76, 3277 (1996).

[37] G. Appelquist et al., Phys. Rev. Lett. 76, 3907 (1996).

[38] T. A. Armstrong et al., Phys. Rev. Lett. 79, 3612 (1997).

[39] C. Greiner and B. Müller, Phys. Lett. B 219, 199 (1989).

[40] D. Anchishkin, U. Heinz, and P. Renk, Phys. Rev. C 57, 1428 (1998).

[41] F. Wang and S. Pratt, Phys. Rev. Lett. 83, 3138 (1999).

[42] A. Ohnishi, Y. Hirata, Y. Nara, S. Shinmura, and Y. Akaishi, nucl-th/9903021.

[43] J. P. Coffin and C. Kuhn, J. Phys. G 23, 2117 (1997).

[44] S. D. Paganis et al., nucl-ex/9910007.

[45] C. Greiner, P. Koch, and H. Stöcker, Phys. Rev. Lett. 58, 1825 (1987). 